Relations industrielles

Industrial Relations

\title{
Applications of Graph Theory to Group Structure, Claude
} Flament, Prentice-Hall, Inc., Englewood Cliffs, New Jersey, 1963, 142 pages.

\section{Fernand Blais}

Volume 20, numéro 4, 1965

URI : https://id.erudit.org/iderudit/027624ar

DOI : https://doi.org/10.7202/027624ar

Aller au sommaire du numéro

Éditeur(s)

Département des relations industrielles de l'Université Laval

ISSN

0034-379X (imprimé)

1703-8138 (numérique)

Découvrir la revue

Citer ce compte rendu

Blais, F. (1965). Compte rendu de [Applications of Graph Theory to Group Structure, Claude Flament, Prentice-Hall, Inc., Englewood Cliffs, New Jersey, 1963, 142 pages.] Relations industrielles / Industrial Relations, 20(4), 716-717. https://doi.org/10.7202/027624ar

Tous droits réservés @ Département des relations industrielles de l'Université Laval, 1965
Ce document est protégé par la loi sur le droit d'auteur. L’utilisation des services d'Érudit (y compris la reproduction) est assujettie à sa politique d'utilisation que vous pouvez consulter en ligne.

https://apropos.erudit.org/fr/usagers/politique-dutilisation/ 


\section{RECENSION S BOOKS REVIEW}

Modern Organizations, por Amitai Etziori, Foundations of Modern Sociology Series, Prentice Hall, Inc., Englewood Cliff, New Jersey, 1964, 120 pp

Chaque volume de cette série éditée par le professeur Alex Inkeles tente d'onalyser un aspect particulier de la vie sociale ou faire état de la théorie et de la recherche dons un secteur porticulier de la sociologie. Modern Organizations vient enrichir cette série par une présentation critique de la sociologie des organisations. En décrivant la nature et les buts d'une organisation, l'auteur fait ressortir le dilemne ouquel foit face toute orgonisotion: maintenir un certain degré d'efficacité tout en permettont oux membres qui en font partie de retirer un certain degré de sotisfaction.

L'outeur distingue plusieurs courants de pensée en théorie de I'organisation. Le fayolisme et le toylorisme constituent la théorie classique qui est un ensemble de principes dont l'application vise surtout à assurer l'efficacité administrative. L'école des relations humaines déclenche un mouvement de pensée appuyé sur la recherche empirique et tente de démontrer les limites de l'approche classique en mettont l'accent sur les conditions qui permettent l'obtention d'un certain degré de satisfaction et d'harmonie au sein des organisations.

L'école «structuraliste développe un courant théorique en réaction contre les deux tendances précédentes et fait ressortir toute la nature du dilemme en montrant un conflit possible entre la réalisation des buts de l'organisation et celle des aspirations personnelles des membres qui la composent. Nous retrouvons dans ce même courant de pensée les modèles de Weber qui tentent de reproduire l'aspect formel des organisations.

Le problème du contrôle au sein des organisations constitue oussi un autre courant de pensée. L'auteur réussit à élaborer une typologie des organisations en prenant comme point de référence les formes de contrôle qui sont exercées

L'incompatibilité entre l'autorité hiérarchique et l'autorité \& professionnelle » (celle qui se fonde sur la connaissance spécialisée) ne fait qu'accentuer le dilemme dont on a décrit la nature ou début. L'auteur donne un compte rendu des trovaux récents traitant des difficultés que présente l'utilisation des experts au sein de la gronde organisation.

Uu neuvième chapitre traitant des relotions qui existent entre le pouvoir de l'organisction et le contrôle que détient le consommateur. Les relations entre l'organisation et son milieu ambiant dans divers types de société constituent l'objet du dernier chapitre. Comme la sociologie des organisations est en train de se constituer, il a été difficile pour l'auteur de conserver une certoine unité ou un thème commun dans so présentation. Cependont, le volume constitue un exposé très succinct qui nous permet de saisir rapidement l'état actuel de la connoissance dans ce secteur de la sociologie.

\section{Laurent Bëlanger}

\section{Applications of Graph Theory to Group \\ Structure, Claude Flament, Prentice-Hall, Inc., Englewood Cliffs, New Jersey, 1963. 142 poges. \\ Ce volume fait figure de pionnier dons les opplicotions d'une théorie mathématique des relations orbitraires au chomp des sciences du comportement}

Le problème de réduire le comportement humain en termes mathématiques nécessite l'établissement d'un parallèle entre le comportement et une structure mathématique démontrable. La théorie des graphes fournit ainsi un véhicule idéal pour les structures riches en possibilités d'applicotions aux sciences du comportement et cette étude explore les sources de ce domaine et développe plusieurs exemples importants.

On croit souvent que la théorie des graphes ne peut-être employée que pour des descriptions statiques; cela n'est pas le cas comme on le remarque au chapitre 3 . alors que l'auteur traite du \& balancing processus"

La première section est une introduction à lo théorie. Une discussion des canqux de communications suit cette introduction 
Enfin, la section trois aborde le processus de balancement.

Cette monographie apporte donc une contribution importante à ce champ de recherche et donne un compte-rendu des explorations et résultots obtenus jusqu'ici.

\section{Fernand Blais}

Emplois et gains dans les professions scientifiques et techniques, 1959-1962, Les travailleurs intellectuels: rapport no 14, Hon. Allan MacEachen, George V, Haythorne, Direction de I'Economique et des Recherches, Ministère du Travail, Ottawa, octobre 1964, 26 pages.

Ce rapport du Ministère fédéral du travail donne les gains et l'emploi dans les professions scientifiques et techniques, de 1959 à 1962 , et vient compléter une série de données publiées antérieurement dans le rapport no 12, de la même série, sur les travailleurs intellectuels (ovant-propos).

Recueillies par échontillonnage, des données sur l'instruction, l'emploi et les gains sont disponibles depuis 1958. Elles fournissent des renseignements sur le marché du travail dans six domaines de spécialisations: l'agriculture, l'architecture, le génie, les sciences forestières, les sciences naturelles et la médecine vétérinaire. Le rapport est centré davantage, cependant, sur le génie et les sciences seulement, mais les renseignements conservent six variables: l'âge, l'instruction, le nombre d'années depuis le boccolouréot, le genre d'employeur, la région d'emploi et la fonction. (p. 5)

\section{Fernand Blais}

\section{Poor Countries and Authoritarian Rule,} Maurice F. Neufeld, New York State School of Industrial and Labor Relations, Cornell University, Ithaca, New York, 1965, 240 pages.

Les pays pauvres connoissent des problèmes économiques difficiles. Ils possèdent de faibles taux de croissance et le fossé avec les poys riches s'accentue toujours. L'ignorance de ces peuples demande aussi des transformations radicales.

Lo situation est urgente et les leaders politiques qui ont pris le pouvoir ovec la promesse d'une perspective économique meilleure souffrent, en courte période, des reproches de lo population.
Pour foire face à ces reproches, ces derniers ont tendance à délaisser la forme de gouvernement démocratique pour s'orienter vers un grouvernement autoritaire, en développant un système politique ò parti unique et en exerçant une coercition gouvernementole très poussée.

II va sans dire que ces changements vont influencer toute la vie socio-économique de ces pays de même que les relations internationales pour le reste du siècle.

Ainsi, Neufel a développé neuf propositions qui tentent d'expliquer le cheminement de ces pays vers l'état autoritaire sinon dictatorial. Il a commencé à. développer ces propositions lors de son étude: \& Italy: School for Awokening Countries », bien qu'ò ce moment là elles étaient peu clarifiées. Aujourd'hui, il développe ces propositions dans des chapitres différents, traitant ainsi de la liberté, du nationalisme, du syndicalisme, des gouvernements et des relations extérieures.

Certains diront que I'histoire de ces pays conquis d̀ l'indépendance est relativement jeune. Mais elle est déià assez longue pour expliquer le développement de certaines formes autocratiques dans les gouvernements. D'ailleurs l'auteur explique ce développement, par ses neuf propositions, comme un processus continu et quasi inévitable, à moins d'un renversement inattendu dans le domaine économique. Toutefois, ces propositions s'appliquent à des degrés différents selon les pays et selon des circonstances, tout en étant un reflet assez fidèle de la situation de ces pays.

\section{Fernand Blais}

\section{Concentration in the Manufacturing Indus-} tries of the United States, Ralph L. Nelson, A Midcentury Report - New Haven and London, Yale University Press, 1963, 288 pages.

$1 /$ peut paraître inévitable que le contrôle d'une industrie soit dans les mains de quelques-uns à un moment ou l'autre de son évolution. La monographie, por Nelson, sur la concentration dans l'industrie manufacturière aux Etats-Unis, révèle justement que cette concentration varie dans le temps.

Cette étude faite à partir de données non-publiées du Bureau de la Statistique, tente de conserver le problème des monopoles dans un contexte adéquat, de même qu'elle fournit des renseignements sur le processus de concentration et son influence sur le modèle de croissance de cette industrie. 